\title{
Low power hybrid PG_Filter- AGC analog baseband for wireless receivers
}

\author{
Yongqian Du${ }^{1}$, Wei $\mathrm{Hu}^{2 \mathrm{a})}$, Gui-fang $\mathrm{Li}^{1}$, and Shibin $\mathrm{Liu}^{1}$ \\ ${ }^{1}$ School of Electronics and Information, Northwestern Polytechnical University, \\ Xi'an, 710072, P. R. China \\ ${ }^{2}$ Department of Microelectronics Science and Engineering, Fuzhou University, \\ Fuzhou, Fujian, 350116, P. R. China
}

a)whu@fzu.edu.cn

\begin{abstract}
A low power hybrid PG_Filter-AGC analog baseband is presented, including a programmable filter $\left(P G_{-}\right.$filter) and an auto gain control core (AGC_core). It adopts the digital-plus-analog mixed gain control methodology, resulting in an effective power reduction and a decibel gain error improvement. To further reduce the power of the AGC_core, a low power Variable Gain Amplifier (VGA) adopting sub-threshold design methodology is presented. Furthermore, a self-adaptive threshold voltage compensation (SATC) scheme is proposed to guarantee the good anti-process variation performance for sub-threshold design methodology. The hybrid analog baseband has been fabricated under SMIC $0.18 \mu \mathrm{m}$ CMOS process, with a die size of $0.45 \mathrm{~mm}^{2}$, where the $A G C_{-}$core occupies an area of $0.28 \mathrm{~mm}^{2}$. The test results demonstrate a total power of $4.1 \mathrm{~mW}$, where the $A G C_{\text {_ccore }}$ consumes a power of $0.39 \mathrm{~mW}$. A consecutive gain dynamic range of $80 \mathrm{~dB}$, with a decibel gain error small than $\pm 0.39 \mathrm{~dB}$, is achieved and the cutoff frequency ranges from $0.5 \mathrm{MHz} 30 \mathrm{MHz}$.
\end{abstract}

Keywords: low power, analog baseband, sub-threshold, hybrid, AGC Classification: Integrated circuits

\section{References}

[1] B. Malki, et al:: “A $150 \mathrm{kHz}-80 \mathrm{MHz} \mathrm{BW}$ discrete-time analog baseband for software-defined-radio receivers using a 5th-order IIR LPF, active FIR and a 10 bit $300 \mathrm{MS} / \mathrm{s}$ ADC in $28 \mathrm{~nm}$ CMOS," IEEE J. Solid-State Circuits 51 (2016) 1593 (DOI: 10.1109/JSSC.2016.2561979).

[2] S. D'Amico, et al.: "A low-power analog baseband section for $60-\mathrm{GHz}$ receivers in 90-nm CMOS," IEEE Trans. Microw. Theory Techn. 62 (2014) 1724 (DOI: 10.1109/TMTT.2014.2332877).

[3] Y. Wang, et al.: "Highly programmable analog baseband for multistandard wireless receivers in 65-nm CMOS," IEEE Trans. Circuits Syst. II, Exp. Briefs 62 (2015) 296 (DOI: 10.1109/TCSII.2014.2368975).

[4] H. Darabi, et al.: "Highly integrated and tunable RF front ends for programmable multiband transceivers, a tutorial," IEEE Trans. Circuits Syst. I, Reg. Papers 58 (2011) 2038 (DOI: 10.1109/TCSI.2011.2162460). 
Trans. Circuits Syst. I, Reg. Papers 53 (2006) 1648 (DOI: 10.1109/TCSI.2006. 879058).

[6] S. H. Yang and C.-C. Wang: "A 48-dB dynamic gain range/stage linear-in-dB low power variable gain amplifier for direction conversion receivers," IEEE J. Solid-State Circuits 38 (2011) 1602.

[7] C. W. Mangelsdorf: "A variable gain CMOS amplifier with exponential gain control," Symposium on VLSI Circuits. Digest of Technical Papers (2000) 146 (DOI: 10.1109/VLSIC.2000.852875).

[8] H. Lee and S. Mohammadi: "A $3 \mathrm{GHz}$ subthreshold CMOS low noise amplifier," Proc. 2006 IEEE Radio Frequency Integrated Circuits (RFIC) Symposium (2006) 494 (DOI: 10.1109/RFIC.2006.1651199).

[9] H. Lee and S. Mohammadi: "A subthreshold low phase Noise CMOS LC VCO for ultra low power applications," IEEE Microw. Wireless Compon. Lett. 17 (2007) 796 (DOI: 10.1109/LMWC.2007.908057).

[10] Y. Du, et al.: "A reliable demodulator and a power regulation circuit with an accurate and small-size voltage reference in UHF RFID," Microelectronics J. 44 (2013) 72 (DOI: 10.1016/j.mejo.2012.10.008).

[11] Q. Lei, et al:: "A low-power CMOS VGA with 60-dB linearly controlled gain range for GPS application," 2008 IEEE Solid-State and Integrated-Circuit Technology (2008) 1669 (DOI: 10.1109/ICSICT.2008.4734873).

[12] T. Yamaji, et al.: "Temperature-stable CMOS variable-gain amplifier with 80dB linearly controlled gain range," IEEE J. Solid-State Circuits 37 (2002) 553 (DOI: 10.1109/4.997847).

[13] V. Giannini, et al.: "Flexible baseband analog circuits for software-defined radio front-ends," IEEE J. Solid-State Circuits 42 (2007) 1501 (DOI: 10.1109/ JSSC.2007.899103).

[14] B. Razavi: Design of Analog CMOS Integrated Circuits, McGraw-Hill International Edition (2000).

\section{Introduction}

The analog baseband composed of intermediate frequency (IF) filter and Auto Gain Control (AGC) circuit is an indispensable circuit block for wireless receiver. The AGC plays an important role in stabilizing the output power of the receiver and relaxes the required dynamic range of cascaded analog to digital converter (ADC). The IF filter selects desired signals from interferences and anti-aliases unwanted noise and blockers. Both of these two circuits are crucial for excluding unwanted effects, such as the interferences, aliased noise, and gain variation [1].

Power consumption is increasingly becoming the dominant factors and inevitable urgent requirements for wireless receivers, especially when taking into account of the explosive growth of mobile, portable applications [2, 3]. This is also quite an issue for the analog baseband. However, most studies, which tend to require huge power, do not pay sufficient attention to these issues $[3,4]$.

In this work, a low power hybrid PG_filter-AGC analog baseband is proposed. By adopting a hybrid architecture, a digital-plus-analog mixed gain control methodology is achieved. Then, the required gain dynamic range of $A G C_{-}$core is relaxed. As a result, a less gain of the $A G C_{-}$core is required, and the power of the analog baseband is reduced from the system level design. Moreover, a less gain dynamic range also results in an improved decibel gain error of $A G C_{-}$core. 
For the $A G C_{-}$core, the necessary decibel linear gain control characteristic of VGA is crucial for stabilizing the settling time of the AGC loop. For full-CMOS implementation of VGA, typical approaches includes the digital or analog design strategy. The digital solutions usually adopts switchable resistor network [5], while the analog solutions include the Taylor series approximation [6] or the pseudoexponential approximation [7]. However, these solutions suffer from high-power and large decibel gain error, especially when a large gain dynamic range is required. These defects greatly restrict their application prospect when facing the urgent low power requirement.

With the progress of the semiconductor technology and design strategy, the sub-threshold design methodology, which demonstrates a well exponential I-V curvature, has been verified as a realistic low-power solution. Lee [8] has verified the reliability of the sub-threshold low noise amplifier (LNA) and the feasibility of the sub-threshold voltage controlled oscillator (VCO) [9]. A sub-threshold voltage reference is proposed by $\mathrm{Du}[10]$ with satisfactory performance. The sub-threshold design methodology also provides a new low power solution for VGA.

In this work, a low power VGA adopting sub-threshold design methodology is proposed. Furthermore, a self-adaptive threshold compensation (SATC) methodology is introduced to guarantee a good anti-process variation performance for the sub-threshold design methodology.

The hybrid analog baseband has been fabricated in SMIC $0.18 \mu \mathrm{m}$ CMOS process. Test results demonstrate a dynamic gain rang of $80 \mathrm{~dB}$ with a decibel gain error small than $\pm 0.39 \mathrm{~dB}$, while the cut-off frequency ranges from $0.5 \mathrm{MHz} \sim$ $30 \mathrm{MHz}$. The total power of the hybrid analog baseband is $4.1 \mathrm{~mW}$, where the $A G C \_$core consumes a power of $0.39 \mathrm{~mW}$.

\section{System architecture}

The proposed analog baseband shown in Fig. 1 adopts a hybrid architecture, where the $P G_{-}$filter, whose gain is digitally programmable, is subsumed into the AGC gain control loop and acts as the gain stage of the VGA. As a result, by cascading the $P G_{-}$filter and a VGA with an analog consecutive controllable gain, a digitalplus-analog mixed gain control strategy is achieved. Then, even with a small gain dynamic range of VGA, a large gain dynamic rang of the analog baseband is

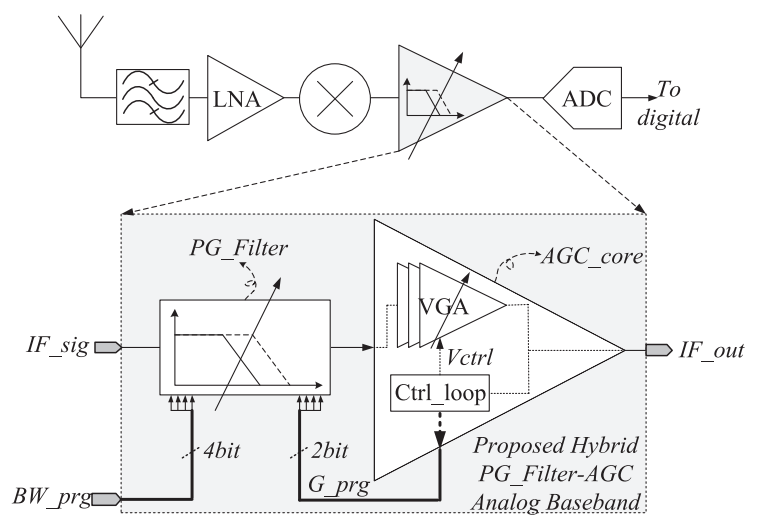


achieved, and the decibel gain error is only determined by the VGA, as shown in Fig. 2. In this work, the gain dynamic range of the VGA is $23 \mathrm{~dB}$ (including a $3 \mathrm{~dB}$ redundant gain for gain variation suppression). Combined with the $P G_{-}$filter, the gain step of which is set to be $20 \mathrm{~dB}$, a gain dynamic range of $80 \mathrm{~dB}$ of the analog baseband is achieved.

With the above architecture design methodology, a less gain dynamic range of VGA is required, which means less gain stages of VGA. Consequently, the power and hardware cost of the VGA is greatly reduced. Moreover, a smaller dynamic gain range of the VGA also means a smaller decibel gain error. With the hybrid digital-plus-analog mixed gain control strategy, the reduction of the gain dynamic range of VGA also helps reducing the decibel gain error of the analog baseband, as the decibel gain error is determined by the VGA, which is also shown in Fig. 2.

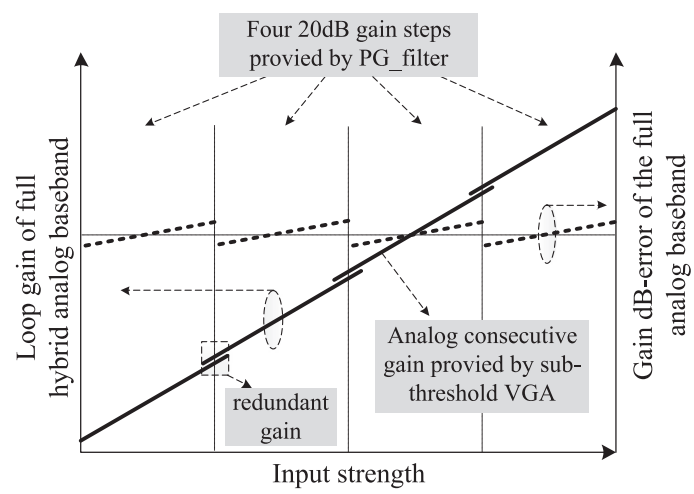

Fig. 2. Scheme of the digital-plus-analog mixed gain control strategy

\section{Detailed circuit design of hybrid analog baseband}

\section{A. The VGA adopting sub-threshold design methodology a) Architecture design}

Fig. 3(a) shows the configuration of the proposed VGA. The sub-threshold exponential current generator (Iexp_gen) and the SATC block form the sub-threshold decibel gain control circuit to realize decibel gain for VGA. There are two gain stages for the VGA and each gain stage is comprised of two sub-amplifiers. The gain characteristic of the sub-amplifier is shown in the upper-left side of Fig. 3(a). When the exponential I-V function between $V_{\text {cg }}$ and $I_{\text {exp }}$ holds, the gain of the VGA demonstrates a good decibel gain characteristic. Then, how to achieve the exponential I-V function between $V_{\text {cg }}$ and $I_{\exp }$ is crucial.

Mexp, the core transistor of Iexp_gen is biased in the sub-threshold region. According to the well-established exponential I-V curvature of sub-threshold MOS transistor $[8,9,10]$, the function between $V_{\mathrm{cg}}$ and $I_{\exp }$ can be expressed as:

$$
\begin{gathered}
I_{\exp }=I_{s 0} S_{M \exp } \exp \left(\frac{V_{c g}-V_{t h}-V_{o f f}}{n V_{T}}\right)\left(1-\exp \frac{-V_{d s}}{V_{T}}\right) \\
I_{s 0}=\mu_{0} \sqrt{\frac{q \varepsilon_{\mathrm{si}} N_{c h}}{2 \Phi_{s} V_{T}}}
\end{gathered}
$$

where: $S$ and $V_{\text {th }}$ is the aspect ratio and threshold voltage, $V_{\mathrm{ds}}$ is the drain and source voltage difference. $V_{\mathrm{T}}=k T / q\left(26 \mathrm{mV} @ 27^{\circ} \mathrm{C}\right) . n$ is defined as the differ- 


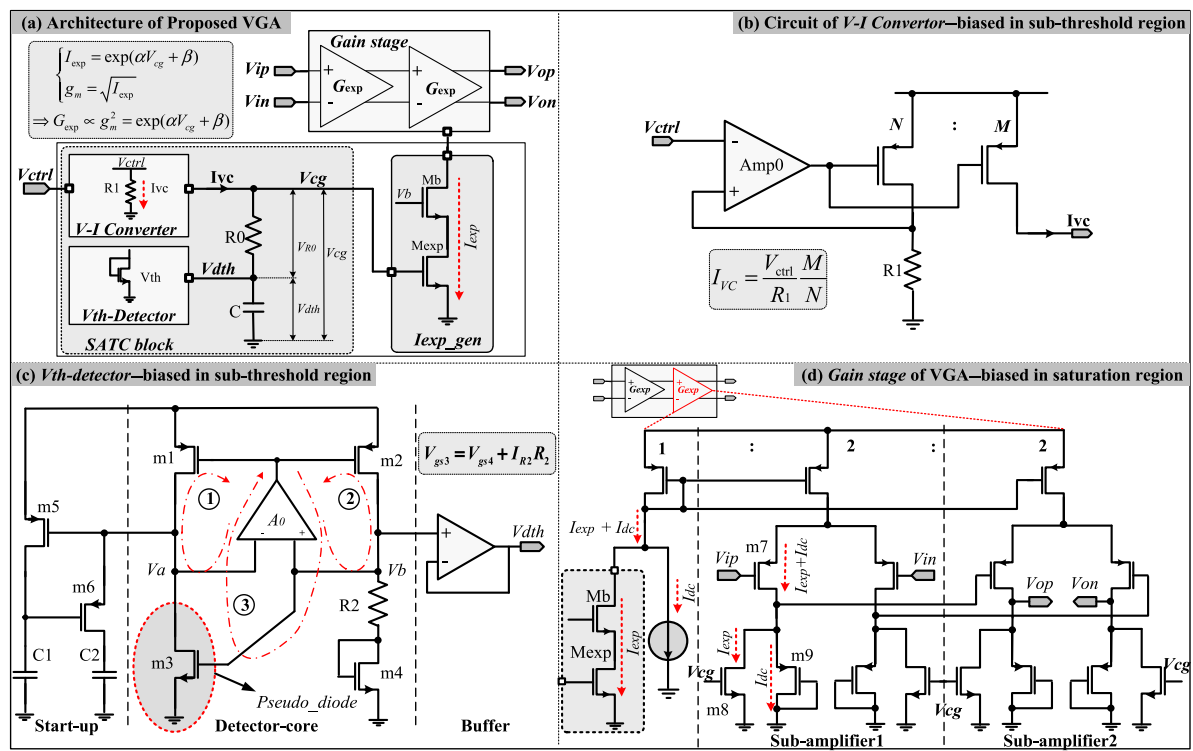

Fig. 3. Full circuit of the proposed VGA

ential of the gate voltage $V_{\mathrm{G}}$ to the cut-off voltage $V_{\mathrm{p}}$, which ranges from 1 to 2 . $V_{\text {off }}$ is the gate-source voltage tested when $I_{\mathrm{ds}}=0$, and for the process adopted in this work, $V_{\text {off }}=130 \mathrm{mV} . \mu_{0}, \varepsilon_{\mathrm{si}}, \Phi_{\mathrm{s}}$ and $N_{\mathrm{ch}}$ are parameters defined by process.

When $V_{\mathrm{ds}}>4 V_{\mathrm{T}}$, the last term in Eq. (1) can be neglected. $I_{\mathrm{s} 0} S_{\mathrm{Mexp}}$ is a constant when design parameters are set, and supposed it equal to $\lambda$, then:

$$
I_{\exp }=\lambda \exp \left(\frac{V_{c g}-V_{t h}-V_{o f f}}{n V_{T}}\right)
$$

Then, the exponential I-V function between $V_{\text {cg }}$ and $I_{\text {exp }}$ holds, and the decibel gain tuning characteristic of the VGA is guaranteed.

The exponential I-V curvature of sub-threshold MOS transistor is crucial for the sub-threshold design methodology. However, as shown in Eq. (3), $I_{\exp }$ shows a direct correlation with $V_{\text {th }}$. The variation of $V_{\text {th }}$ may be bigger than $100 \mathrm{mV}$ or even larger. Thus, two main problems in the sub-threshold design methodology is presented: 1) MOS transistor should be biased in sub-threshold region reliably to guarantee the exponential I-V curvature; 2) the process variation of $V_{\text {th }}$ is of great concern, which may cause a huge variation of $I_{\text {exp }}$ or even drive MOS transistor out of sub-threshold region. Both problems can collapse the performance and even the function of the exponential I-V curvature between $V_{\mathrm{cg}}$ and $I_{\text {exp }}$. However, to the authors' knowledge, few studies reports solutions to these problems.

To address the questions for sub-threshold design methodology, a SATC block shown in Fig. 3(a) is introduced. With the help of the SATC block, which will be proved in the following text, Mexp is biased in sub-threshold region reliably. Moreover, the process variation of $V_{\text {th }}$ is simultaneously cancelled, which guarantees a stable exponential I-V curvature between $V_{\mathrm{cg}}$ and $I_{\exp }$. Finally, a stable decibel gain of the VGA even under huge process variation is guaranteed.

\section{b) SATC scheme and circuit design}

The schematic of the V-I converter is shown in Fig. 3(b). Then, the voltage difference across the gate and source of $M \exp , V_{\text {cg }}$, can be deduced as: 


$$
V_{c g}=V_{g s, M \exp }=\frac{M}{N} \frac{R_{0}}{R_{1}} V_{c t r l}+V_{d t h}
$$

where, $V_{\text {dth }}$ generated by the Vth-detector is a crucial parameter for SATC scheme, as proved in the following formulas.

The schematic of the Vth-detector is shown in Fig. 3(c). Transistor $\mathrm{m} 1 \sim \mathrm{m} 4$ are all biased in the sub-threshold region, and transistor $\mathrm{m} 3$ works as a pseudo-diode. Combined with amplifier $A_{0}$, three feedback loops as shown in Fig. 3(c) are achieved, the total gain of which forces $V_{\mathrm{a}} \approx V_{\mathrm{b}}$, and this is the base for the following deduction. It should be note that, the introduction of the pseudo-diode helps to improve the total loop gain of the Vth-detector. This helps $V_{\mathrm{a}}$ and $V_{\mathrm{b}}$ equal to each other more accurately, as proved in Appendix $\boldsymbol{A}$.

Then, the detected threshold voltage is:

$$
V_{\mathrm{dth}}=V_{\mathrm{th}}+V_{\text {off }}+n V_{\mathrm{T}} \ln \left[\frac{n V_{\mathrm{T}} S_{1}}{R_{2} S_{2} S_{3} I_{\mathrm{s} 0}} \ln \left(\frac{S_{1}}{S_{2}} \frac{S_{4}}{S_{3}}\right)\right]
$$

When $V_{\text {cg }}$ is applied on Mexp, $I_{\exp }$ can then be expressed as:

$$
I_{\exp }=\lambda \exp \left\{\ln \left[\frac{n V_{\mathrm{T}} S_{1}}{R_{2} S_{2} S_{3} I_{\mathrm{s} 0}} \ln \left(\frac{S_{1}}{S_{2}} \frac{S_{4}}{S_{3}}\right)\right]+\frac{M}{N} \frac{R_{0}}{R_{1} n V_{T}} V_{\text {ctrl }}\right\}
$$

The first term in the exponential function and the coefficient of $V_{\text {ctrl }}$ are denoted as $\alpha$ and $\beta$ respectively, then:

$$
I_{\exp }=\lambda \exp \left(\alpha+\beta V_{c t r l}\right)
$$

As long as the design parameters are set, $\alpha, \beta$ and $\lambda$ are all constants. Then, Eq. (7) shows that $I_{\exp }$ is only determined by control voltage $V_{\text {ctrl }}$, while $V_{\text {th }}$ is cancelled by $V_{\mathrm{dth}}$. Moreover, when all design parameters are reasonably set to make:

$$
\frac{M}{N} \frac{R_{0}}{R_{1}} V_{c t r l}+\alpha n V_{T}+V_{o f f}<0
$$

then, $V_{\text {cg }}=V_{\mathrm{gs}, \text { Mexp }}<V_{\text {th }}$ will always holds, Mexp is always biased in subthreshold region reliably, even with a large process variation. Moreover, the variation of $I_{\exp }$ due to $V_{\text {th }}$ drift is also compensated, which can be seen from Eq. (3) and (7).

Based on the above analysis, the problems of sub-threshold design methodology mentioned above are successfully solved, which guarantees good performance and robustness of the proposed sub-threshold decibel gain control circuit.

\section{c) Gain stage design}

To realize the gain stage with a gain characteristic as shown in upper-left side of Fig. 3(a), the most simple and direct way is adopting two cascaded sub-amplifiers with resistive load, as shown in Fig. 4. However, under the bias of a changing exponential current for gain tuning, the output quiescent output voltage of each subamplifier is: $V_{1}$ (or $\left.V_{\text {op }}\right)=R I_{\text {exp }}$. As a result, the gain controlled by tuning $I_{\exp }$ will result in a huge deviation of the quiescent operation point of the sub-amplifier. This is of great danger, which may drive the sub-amplifier into linear or off region. As a result, the allowable operating range is restricted, and the gain dynamic range of the VGA is also narrowed, as shown on the right side of Fig. 4. 
To stabilize the quiescent operation point of the sub-amplifier, a current network loaded sub-amplifier is proposed, as shown in Fig. 3(d). It is composed of a diode connected MOS transistor and a current source biased by $I_{\exp }$. Then, the output quiescent operation point of each sub-amplifier can be re-written as:

$$
V_{1}\left(\text { or } V_{o p}\right)=\sqrt{\frac{2 I_{d c}}{\mu_{n} C_{o x} S_{9}}}+V_{t h}
$$

Thus, the output quiescent operation point of each sub-amplifier remains stable, even under a large variation of the exponential current $I_{\text {exp }}$ for gain tuning.

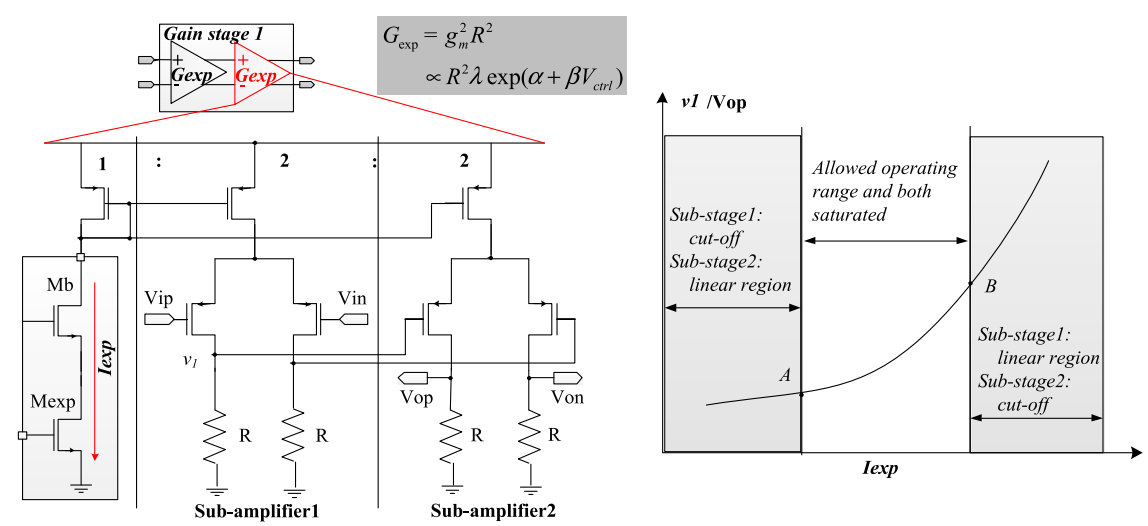

Fig. 4. Schematic of sub-amplifier of VGA and the narrowed operating range

According to Fig. 3(d), with reasonable parameters setting, the gain of each gain stage can be derived as:

$$
G_{\text {exp }}=A_{\text {sub-amplifier }}^{2}=\left(\frac{g_{m 7}}{g_{m 9}}\right)^{2}=\frac{S_{m 7}}{S_{m 9}}\left[1+\frac{\lambda}{I_{d c}} \exp \left(\alpha+\beta V_{c t r l}\right)\right]
$$

With proper design parameters settings, the constant "1" in the square brackets can be neglected (this approximation may introduce some decibel gain error. Nevertheless, the hybrid architecture of the analog baseband helps to reduce the gain tuning rang of the VGA, and decibel gain error is also reduced to a negligible level as mentioned above), then:

$$
\ln \left(G_{\text {exp }}\right)=\eta+\beta V_{c t r l}, \quad \eta=\alpha+\ln \left(\frac{S_{m 7}}{S_{m 9}} \frac{\lambda}{I_{d c}}\right)
$$

Eq. (11) indicates good decibel gain of the VGA. Moreover, as the $V_{\text {th }}$ variation of $I_{\exp }$ has been compensated by the proposed SATC scheme, the gain of the gain stage of VGA is also only determined by the control voltage $V_{\text {ctrl }}$. Finally, a stable gain of VGA is achieved despite the process variation.

\section{B. Ctrl-loop circuit design}

The loop control circuit Ctrl_loop is shown in Fig. 5, where the Clamp circuit is used to set the highest voltage level of $V_{\text {ctrl }}$ to guarantee Eq. (8) always holds. The Peak_detector senses the highest voltage level of the output of the analog baseband, while the target output amplitude is set by $V_{\text {amp }}$ (the input of the Error amplifier). 


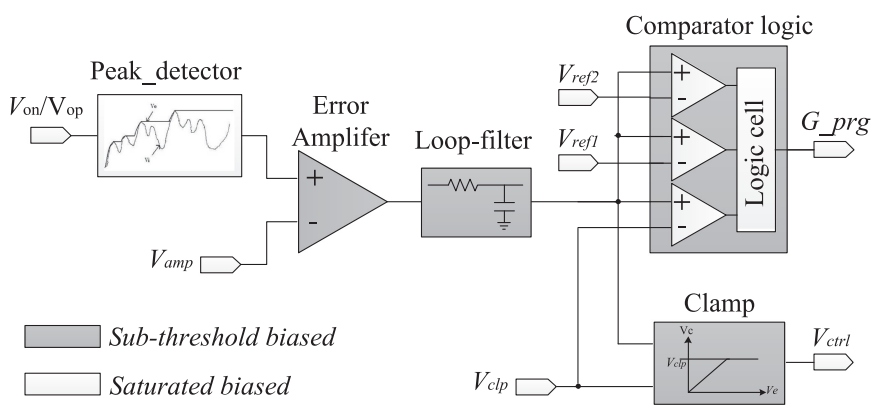

Fig. 5. Architecture of the Ctrl_loop

The Comparator logic generates 4 bits digital control code $G_{-} p r g$, and it is then send into the $P G_{-}$filter to decide the digital gain of the $P G_{-}$filter.

Moreover, the Error amplifier, Clamp circuit and Comparator logic are all biased in the sub-threshold region to save power without sacrificing performance, while the Peak detector is biased in the saturation region for a higher work speed.

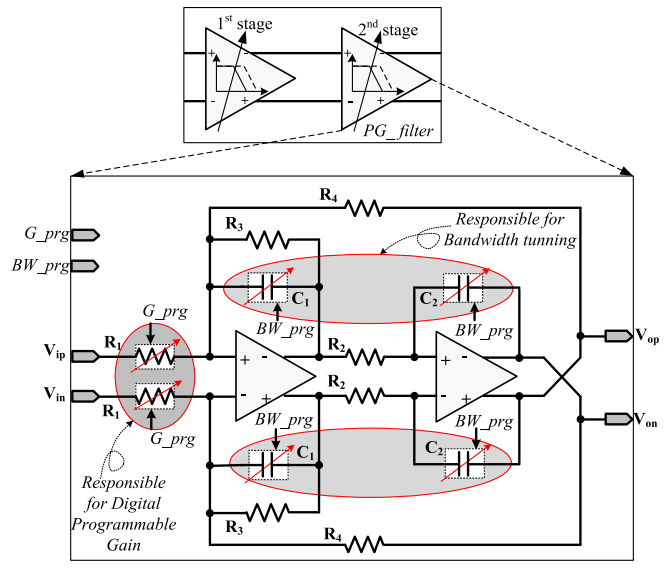

Fig. 6. Circuit design for $P G_{f}$ filter

\section{Citcuit desing of PG_filter}

For $P G_{-}$filter, the Sallen-Key Biquade filter synthesizing method is discarded for its sensitivity to the process variation and the parasitic effect. In this study, the TowThomas Biquade filter synthesizing method is adopted for its relatively high stability and low sensitivity to the parasitic effect and the process variation.

Fig. 6 shows the schematic of the 4th-order butterrworth (which is chosen for its relax requirements on core operational amplifier) low pass $P G_{-}$filter. It has two cascade 2nd-order Tow-Thomas Biquades, with the transfer function expressed as:

$$
H(s)=\frac{\frac{1}{R_{1} R_{2} C_{1} C_{2}}}{s^{2}+\frac{s}{R_{3} C_{1}}+\frac{1}{R_{2} R_{4} C_{1} C_{2}}}
$$

Then, the detail parameters of the Tow-Thomas Biquade including pass-band gain, 3-dB cut-off frequency and $Q$ factor are shown as follows:

$$
A_{0}=\frac{R_{4}}{R_{1}}, \quad f_{c}=\frac{1}{2 \pi \sqrt{R_{2} R_{4} C_{1} C_{2}}}, \quad Q=\frac{R_{3}}{\sqrt{R_{2} R_{4}}} \sqrt{\frac{C_{1}}{C_{2}}}
$$


The methodology of setting the design parameters is of great importance to realize an independent cut-off frequency programming and digital gain control, without changing the $Q$ factor. Then, the following strategy is adopted: tunable resistor $R_{1}$ under the control of $G_{-}$prg is responsible for digital gain control. Adjustment of the product of $C_{1}$ and $C_{2}$ under the control of $B W_{-} p r g$ is responsible for the cut-off frequency manipulating, while $C_{1} / C_{2}$ remains to be a constant and $R_{2}, R_{3}$ and $R_{4}$ remain unchangeable to keep a stable $\mathrm{Q}$ factor.

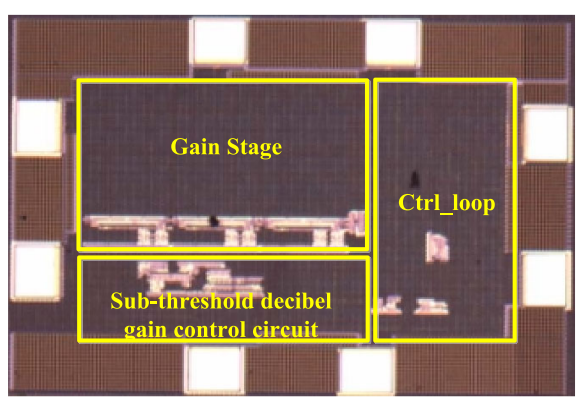

(a)

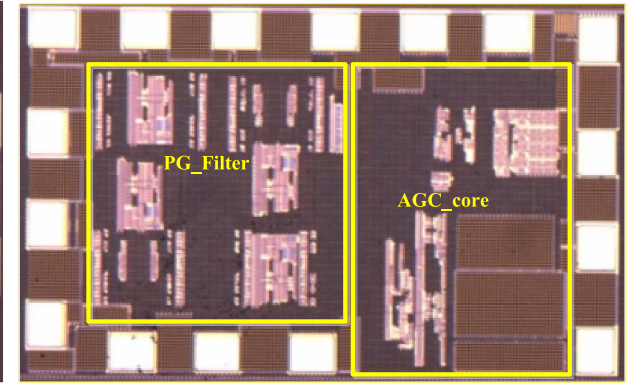

(b)

Fig. 7. Die photo of (a) the independent $A G C_{-}$core; (b) the full hybrid analog baseband

\section{Measurement results}

The analog baseband was fabricated under SMIC $0.18 \mu \mathrm{m}$ CMOS technology. The VGA determins the performance of the analog baseband. Thus, an independent $A G C_{-}$core is taped out and a performance test is setup individually. The photo is shown in Fig. 7(a) with an area of $0.28 \mathrm{~mm}^{2}$, including the Ctrl_loop but without test pads.

Two test modes for AGC_Core are adopted, including an open loop test and a closed loop test: 1) the open loop test is dedicated for the performance of VGA. It is realized by forcing a programmable voltage source on $V_{\text {ctrl }}$. The performance of the proposed VGA is summarized in Table I, which shows much lower power compared with previous works. The test results in Fig. 8 show that the dynamic gain range is $23 \mathrm{~dB}$ while the decibel gain error is less than $\pm 0.39 \mathrm{~dB}$. The test results demonstrate the correct function and good performance of the proposed

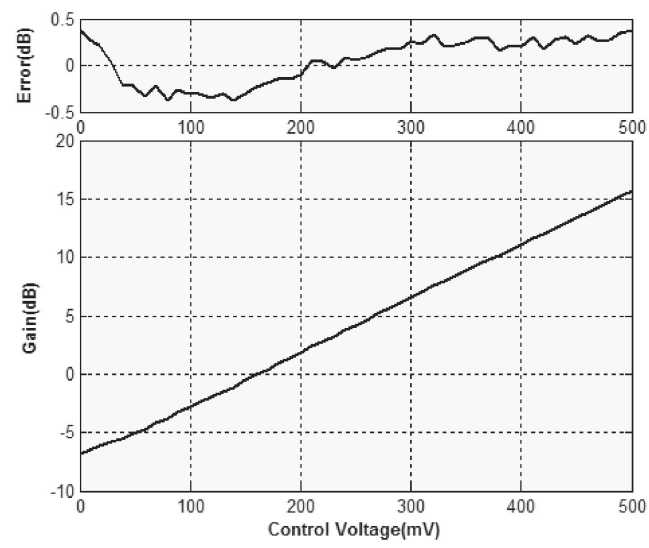

Fig. 8. Gain and $\mathrm{dB}$ error of VGA with respect to $V_{\text {ctrl }}$ 
VGA; 2) A transient closed loop test is then carried out. The test result is shown in Fig. 9, which demonstrate the good performance of the proposed AGC_core.

Table I. Performance of the sub-threshold VGA

\begin{tabular}{l|c|c|c|c|c}
\hline Parameters & {$[5]$} & {$[6]$} & {$[11]$} & {$[12]$} & This Work \\
\hline Power source/V & 1.8 & 1.8 & 1.8 & 2.5 & 1.8 \\
\hline Number of gain stages & 3 & 1 & 3 & 4 & 2 \\
\hline Gain range/dB & 95 & 48 & 60 & 80 & 23 \\
\hline dB-error/dB & NA & NA & NA & \pm 3 & \pm 0.39 \\
\hline Bandwidth/MHz & 32 & 3 & 16 & $30 \sim 210$ & 30 \\
\hline Power/mw & 6.5 & 0.55 & 3.6 & 11 & $0.39^{*}$ \\
\hline
\end{tabular}

*The power listed here is in fact the power of $A G C_{-}$core including the power of the Ctrl_loop.

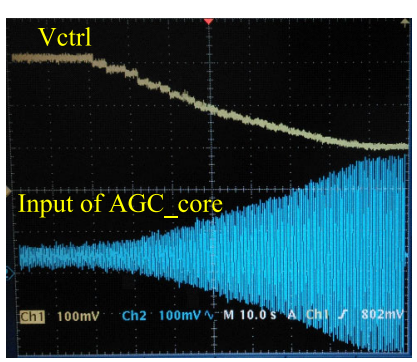

(a)

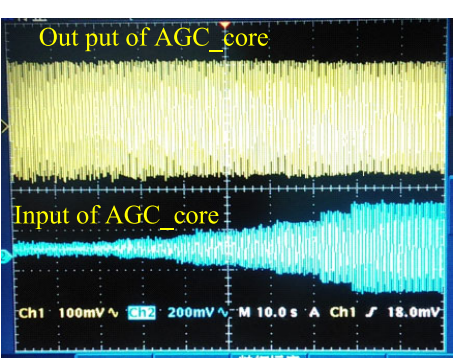

(b)

Fig. 9. Closed loop transient test results of the $A G C_{-}$core

Fig. 7(b) shows the photo of the hybrid analog baseband, occupying a die area of $0.45 \mathrm{~mm}^{2}$ without test pads. The performance summary is shown in Table II. Due to the hybrid architecture, the dynamic gain of the hybrid analog baseband reaches $80 \mathrm{~dB}$, while the $A G C_{-}$core only provides a dynamic range of $23 \mathrm{~dB}$. The total power is $4.1 \mathrm{~mW}$. As compared to former reports, the proposed analog baseband is power and cost efficient.

Table II. Performance of the hybrid analog baseband

\begin{tabular}{|c|c|c|c|c|}
\hline \multicolumn{2}{|c|}{ Parameters } & [3] & [13] & This work \\
\hline \multicolumn{2}{|c|}{ Gain control type } & Digital & Digital & Digital-plus-analog mixed, Consecutively \\
\hline \multicolumn{2}{|c|}{ Power source/V } & 1.2 & 1.2 & 1.8 \\
\hline \multirow{3}{*}{$\begin{array}{l}\text { Maximum } \\
\text { Power/mW }\end{array}$} & PG_filter & - & 21.6 & 3.71 \\
\hline & AGC_core & - & 13.5 & 0.39 \\
\hline & Total & 9.6 & 35.1 & 4.1 \\
\hline \multicolumn{2}{|c|}{ Gain dynamic range/dB } & 72 & 39 & 80 \\
\hline \multicolumn{2}{|c|}{ Cut-off frequency/MHz } & $0.2 \sim 20$ & $0.35 \sim 23.5$ & $0.5 \sim 30$ \\
\hline \multicolumn{2}{|l|}{ Technology } & $65 \mathrm{~nm}$ & $0.13 \mu \mathrm{m}$ & $0.18 \mu \mathrm{m}$ \\
\hline \multicolumn{2}{|c|}{ Circuit Area $/ \mathrm{mm}^{2}$} & 0.8 & 1.56 & 0.45 (AGC_core: 0.28 , including the Ctrl_loop) \\
\hline
\end{tabular}

The cut-off frequency of the analog baseband is shown in Fig. 10(a), which demonstrates a programmable bandwidth ranging from $0.5 \mathrm{MHz}$ to $30 \mathrm{MHz}$. The overall gain characteristic of the full analog baseband with the respect of input 
frequency is shown in Fig. 10(b). Fig. 11 gives the measured closed loop gain and $\mathrm{dB}$ error of the full hybrid analog baseband circuits with respect to $V_{\text {ctrl }}$, which shows the monotonically increasing of the gain with respect to the reduction of the input signal strength. Moreover, the $\mathrm{dB}$ error test result (which is smaller than $\pm 0.39 \mathrm{~dB}$ ) also demonstrates that, the same as depicted in Fig. 2, the hybrid digitalplus-analog mixed gain control strategy of this work helps to improve the decibel gain error of the full hybrid analog baseband.

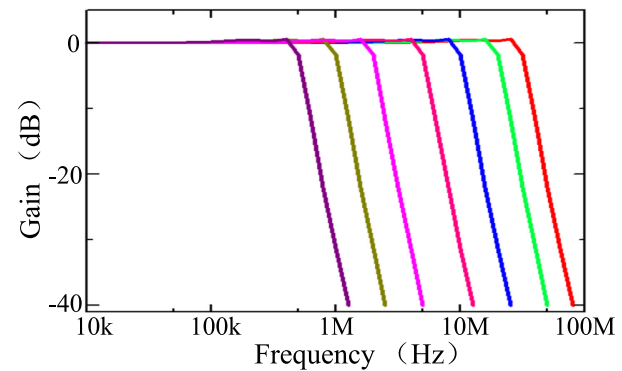

(a)

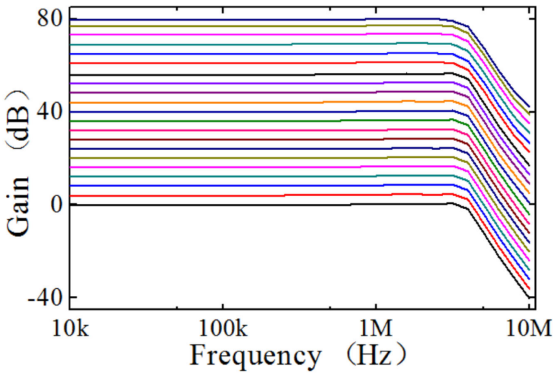

(b)

Fig. 10. (a) Measured cut-off frequency response of the full analog baseband; (b) Measured gain response of the full analog baseband

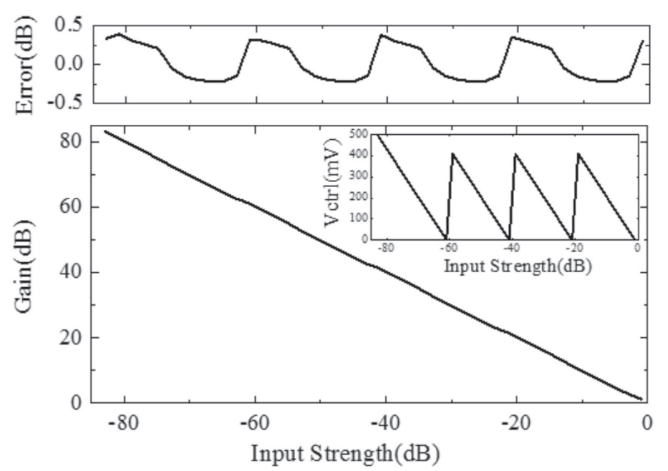

Fig. 11. Gain and $\mathrm{dB}$ error of the full hybrid analog baseband circuits with respect to $V_{\text {ctrl }}$

\section{Conclusion}

A low power hybrid analog baseband is proposed in this study. By adopting a digital-plus-analog mixed gain control methodology, the gain dynamic range of the VGA is relaxed. Thus, the power of the analog baseband is optimized at the system level. Moreover, with a reduced gain tuning range of the VGA, the decibel gain error of the full analog baseband is also improved. For the $A G C_{-}$core, the subthreshold design methodology is adopted for power reduction, while the process variation of sub-threshold design methodology is compensated by a SATC scheme. Moreover, a pseudo diode helping in enhancing the loop gain is introduced to improve the performance of the SATC scheme, while a current network loaded amplifier for the gain stage of VGA is proposed to stable the quiescent operating point. The test results demonstrate a total power dissipation of $4.1 \mathrm{~mW}$, while the $A G C_{\text {_ccore dissipates }} 0.39 \mathrm{~mW}$. The total gain dynamic range of the full analog baseband is $80 \mathrm{~dB}$ with a $\pm 0.39 \mathrm{~dB}$ decibel gain error. 


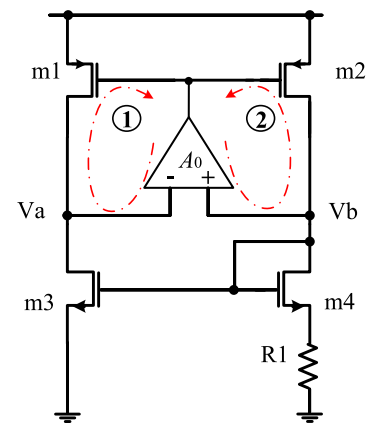

Fig. 12. Schematic of the prototype of Vth-detector

\section{Appendix A: Improvement of the loop gain of Vth-detector}

The prototype of Vth-detector is a sub-threshold $I_{\mathrm{PTAT}}$ current generator illustrated in Fig. 12 [14]. The calculation foundation for this circuit is the assumption of $V_{\mathrm{a}}=V_{\mathrm{b}}$. How $V_{\mathrm{a}}$ and $V_{\mathrm{b}}$ approache to each other is crucial. For the traditional design as shown in Fig. 12, this assumption is guaranteed by the positive feedback loop 1 and the negative feedback loop 2. Thus, the total loop gain is:

$$
L G=g_{m 2}\left[r_{o 2} \|\left(R_{1}+\frac{1}{g_{m 4}}\right)\right] A_{0}-g_{m 1}\left(r_{o 1} \| \frac{1}{g_{m 3}}\right) A_{0}
$$

where: $r_{\mathrm{o}}$ is the small signal resistance of MOS transistor, and $g_{\mathrm{m} 3}=g_{\mathrm{m} 4}, g_{\mathrm{m} 1}=$ $g_{\mathrm{m} 2}$.

When $r_{\mathrm{o}} \gg 1 / g_{\mathrm{m}}$ and $r_{\mathrm{o}} \gg R_{1}$, the loop gain falls into the range of $g_{\mathrm{m}} r_{\mathrm{o}} A_{0}$.

$V_{\mathrm{a}}$ accurately equal to $V_{\mathrm{b}}$ is crucial to the precision of the detection of $V_{\text {th }}$ and for the performance of the SATC scheme. Thus, a higher loop gain is essential.

To improve the loop gain, a pseudo diode connected transistor similar to our previous study is introduced, as shown in the grey block in Fig. 3(c). As a result, a new additional negative feedback path marked as loop 3 in Fig. 3(c) is introduced. Then, the total loop gain can then be re-expressed as:

$L G=g_{m 2}\left[r_{o 2} \|\left(R_{2}+\frac{1}{g_{m 4}}\right)\right] A_{0}-g_{m 2}\left[r_{o 2} \|\left(R_{2}+\frac{1}{g_{m 4}}\right)\right] g_{m 3}\left(r_{o 1} \| r_{o 3}\right) A_{0}-g_{m 1}\left(r_{o 1} \| r_{o 3}\right) A_{0}$

Assume that $r_{\mathrm{o}}$ is much larger than $1 / g_{\mathrm{m}}$, and $R_{2}$, and $g_{\mathrm{m} 3}=g_{\mathrm{m} 4}, g_{\mathrm{m} 1}=g_{\mathrm{m} 2}$, then the loop gain of the proposed Vth-detector can be simplified as:

$$
L G=-g_{m 1}\left(r_{o 1} \| r_{o 3}\right) A_{0}\left[r_{o 2} \|\left(R_{2}+\frac{1}{g_{m 4}}\right) g_{m 3}+1\right] \cong-g_{m 1} g_{m 3}\left(r_{o 1} \| r_{o 3}\right) r_{o 2} A_{0}
$$

It can be concluded that the loop gain when introducing the pseudo diode falls into the range of $\left(g_{\mathrm{m}} r_{\mathrm{o}}\right)^{2} A_{0}$. Thus, the total loop gain is enhanced by $g_{\mathrm{m}} r_{\mathrm{o}}$ times. This will accurately force $V_{\mathrm{a}}=V_{\mathrm{b}}$, which also guarantees the precision of the Vthdetector, and finally, the performance of the SATC is improved.

\section{Acknowledgment}

This work was partly supported by the Fundamental Research Funds for the National Natural Science Foundation of China (Grant No. 61704139 and Grant No. 61504107), Natural Science Foundation of Shaanxi Provincial (Grant No. 2017JQ6051 and Grant No. 2017JM6088), Central Universities of China (Grant No. 3102015ZY043 and Grant No. 3102017zy016). 